Aerospace Science and Technology,

Volume 77, June 2018, Pages 320-331

DOI:10.1016/j.ast.2018.03.013

\title{
Identification of the formation of resonant tones in compressible cavity flows
}

\author{
David Bacci, Alistair J. Saddington ${ }^{1}$, Derek Bray \\ Aeromechanical Systems Group, Centre for Defence Engineering, \\ Cranfield University, Defence Academy of the UK, Shrivenham, SN6 8LA, UK
}

\begin{abstract}
Identification of the fluid dynamic mechanisms responsible for the formation of resonant tones in a cavity flow is challenging. Time-frequency non-linear analysis techniques were applied to the post-processing of pressure signals recorded on the floor of a rectangular cavity at a transonic Mach number. The results obtained, confirmed that the resonant peaks in the spectrum were produced by the interaction of a carrier frequency (and its harmonics) and a modulating frequency. High-order spectral analysis, based on the instantaneous wavelet bi-coherence method, was able to identify, at individual samples in the pressure-time signal, that the interaction between the fundamental frequency and the amplitude modulation frequency was responsible for the creation of the Rossiter-Heller tones. The same technique was also capable to correlate the mode switching phenomenon, as well as the deactivation of the resonant tones during the temporal evolution of the signal.
\end{abstract}

\begin{tabular}{|c|c|c|c|}
\hline \multicolumn{2}{|c|}{ Nomenclature } & \multirow{3}{*}{$\begin{array}{l}\gamma \\
\delta \\
\mu\end{array}$} & \multirow{3}{*}{$\begin{array}{l}\text { modulation parameter } \\
\text { boundary layer thickness }[\mathrm{mm}] \\
\text { mean value }\end{array}$} \\
\hline & & & \\
\hline$C_{p}$ & pressure coefficient & & \\
\hline$D$ & cavity depth $[\mathrm{m}]$ & $\sigma$ & root mean square \\
\hline$f$ & frequency $[\mathrm{Hz}]$ & $\omega$ & angular velocity $\left[\operatorname{rads}^{-1}\right](2 \pi \cdot f)$ \\
\hline$f_{n}$ & Nyquist frequency $[\mathrm{Hz}]\left(f_{s} / 2\right)$ & $\bar{x}$ & time averaged value of the discrete $t$ \\
\hline$f_{r}$ & frequency resolution $[\mathrm{Hz}]$ & & $x_{n}\left(\sum_{j=1}^{N} x_{j} / N\right)$ \\
\hline$f_{s}$ & sampling frequency $[\mathrm{Hz}]$ & $\hat{X}$ & discrete Fourier transform of the var \\
\hline$i$ & imaginary unit & $X^{*}$ & complex conjugate of the variable $X$ \\
\hline$L$ & cavity length $[\mathrm{m}]$ & $\left\langle x_{n}\right\rangle$ & expected value of the discrete time s \\
\hline$M$ & $\begin{array}{l}\text { Mach number, characteristic modes of the signal } \\
\text { in the Strouhal number domain }\end{array}$ & & $x_{n}\left(\sum_{j=1}^{N} x_{j} / N\right)$ \\
\hline$m_{n}$ & nth Rossiter-Heller mode & Abbrevi & tions \\
\hline$p$ & static pressure $[\mathrm{Pa}]$ & $\mathrm{BC}$ & bi-coherence \\
\hline$q$ & dynamic pressure $[\mathrm{Pa}]$ & $\mathrm{BP}$ & bi-phase [rad] \\
\hline St & Strouhal number $\left(f \cdot L / U_{\infty}\right)$ & DFT & discrete Fourier transform \\
\hline$T$ & temperature $[\mathrm{K}]$ & FFT & fast Fourier transform \\
\hline$U$ & flow speed $\left[m s^{-1}\right]$ & FPL & fluctuating pressure level $[\mathrm{dB}]$ \\
\hline$W$ & cavity width $[\mathrm{m}]$ & GWSP & global wavelet spectral power $[\mathrm{dB}]$ \\
\hline
\end{tabular}

Email address: a.j.saddington@cranfield.ac.uk (Alistair J. Saddington)

${ }^{1}$ Corresponding author. 


\begin{tabular}{llll} 
HOSA & high order spectral analysis & SPL & sound pressure level $[\mathrm{dB}]$ \\
IWBC & instantaneous wavelet bi-coherence & STFT & short-time Fourier transform \\
OAFPL & overall fluctuating pressure level $[\mathrm{dB}]$ & WAVT & wavelet average variance trend $[\mathrm{dB}]$ \\
OASPL & overall sound pressure level $[\mathrm{dB}]$ & WSP & wavelet spectral power $[\mathrm{dB}]$ \\
PSD & power spectral density & \multicolumn{2}{l}{} \\
RCS & radar cross section & $\infty$ & freetsream conditions
\end{tabular}

\section{Introduction}

The requirement for modern aircraft to have a reduced radar-cross-section (RCS), as well as improved aerodynamic performance, has introduced the need to incorporate weapon bays in the designs of the next generation of military aircraft. When the bay doors open, the flow in the weapon bay becomes highly turbulent and unsteady, posing a hazard to any desired weapon release. Moreover, airframe and weapon can be damaged by the intense acoustic field that is developed by the flows, which can produce unsteady pressure levels of up to $170 \mathrm{~dB}$ at particular resonant modes. As shown by Rockwell and Naudasher [1], these phenomena are strongly dependant on the geometry as well as on the free-stream conditions. The frequencies, at which the resonance occurs, called Rossiter modes (from the pioneering work of Rossiter [2]), can be estimated, in compressible flow, using Heller and Bliss's semi empirical method [3]. Both the Rossiter and Heller methods were developed for rectangular cavities; however, these equations cannot predict the distribution of the modal amplitudes, which vary greatly depending on the flow condition and cavity geometry. Previous experiments relating to rectangular cavities of various depths have been conducted to study the unsteady fluctuations and to understand the physics behind the phenomenon. Tracy and Plentovich [4] correlated the effect of geometrical parameters and Mach number to the sound pressure level (SPL) and overall sound pressure level (OASPL) distributions inside the cavity, while Ahuja and Mendoza [5] studied the effect of other parameters such as the boundary layer thickness at the cavity's leading edge.

Whilst the basic assumption of the Rossiter-Heller theory on resonating cavities is that the phenomenon is statistically stationary and composed of non-harmonic tones, more recent studies have revealed a more complex nature underlying the oscillating mechanism. Kegerise et al. [6] demonstrated that cavity flow is subject to non-statistically-stationary behaviour, in particular to what is called 'mode switching', i.e. a process whereby the dominant energy shifts from one resonant tone to another as the signal evolves in time. Such behaviour was demonstrated by analysing the pressure histories using the time-frequency analysis capabilities of the wavelet transform. Additionally, it was discovered that the flow is also affected by non-linearites, such as quadratic frequency and phase coupling. These features appear in the frequency spectrum as additional peaks, accompanying the main Rossiter-Heller tones. Utilising high-order spectral analysis (HOSA), Kegerise et al. [6] classified such additional tones as the result of quadratic coupling between the principal tones. A subsequent study by Delprat [7] introduced a new model for the resonating mechanism, which explained the additional peaks as well as the main ones. It was observed that the Rossiter-Heller tones could be interpreted as the result of a frequency shift (i.e. an amplitude modulation) of a frequency-modulated wave. Delprat [7] proposed the following equation for a generic Rossiter tone of $n$th order $\left(f_{n}\right)$.

$$
f_{n}=(n-\gamma) \cdot\left[f_{a} \pm k \Delta f\right] \quad n=1,2,3, \ldots
$$

In Equation 1, $f_{a}$ is the fundamental frequency loop of the cavity (also called the 'carrier frequency'), $\gamma$ is the modulation 
parameter, or the ratio of the amplitude modulation frequency $f_{b}$ to $f_{a}, \Delta f$ is the modulating frequency (which usually coincides with the lowest frequency peak in the spectrum), and $k$ is an integer. From this supposition, Delprat [7] derived the pseudo-harmonic approximation. Hence, the Rossiter tones can be deduced from a knowledge of the carrier frequency, the modulating frequency, and the amplitude-modulation frequency.

One significant outcome of this model was the evidence of an amplitude-modulation mechanism of the fundamental frequency that acted as a frequency shifter and generated the non-harmonic Rossiter tones. It was also responsible for the mode switching phenomena with temporal variation of the parameter $\gamma$ [7]. Additionally, a very low frequency component, which in the past was considered the result of background noise, was identified as a true physical phenomenon. Kegerise et al. [6], using bi-spectral analysis observed that the fundamental frequency, $f_{a}$, experienced amplitude modulation at the $\Delta f$ frequency, whilst frequency-modulation behaviour was observed in the time-frequency analysis. Nevertheless, no significant coupling between the Rossiter modes and the $\Delta f$ frequency could be established; the same was true for the frequency shift operated by the amplitude modulator. As pointed out by Delprat [7], that was an expected outcome because frequency-based bi-spectral analysis cannot resolve FM/AM phenomena since no information is given regarding the temporal evolution of the coupling. The spectrogram itself has difficulties in analysing such complex phenomena since it cannot increase the resolution in time without losing resolution in frequency.

In this study, pressure histories were obtained from wind tunnel experiments on a simple rectangular cavity with a length-todepth ratio of five and at a Mach number of 0.81 . Then, using wavelet analysis as the base transform for HOSA methodologies, the non-linear mechanisms proposed by Delprat's model were explored.

\section{Experimental setup}

Experiments were performed in the closed-circuit, ejector-driven, transonic wind tunnel, located at the Defence Academy of the UK at Shrivenham (Figure 1). The tunnel has a working section $500 \mathrm{~mm}$ long, $206 \mathrm{~mm}$ high and $228 \mathrm{~mm}$ wide. The air supply is provided by a Compair L110-10 compressor, dried, and stored in a $34 \mathrm{~m}^{3}$ reservoir. The wind tunnel is designed to operate in the Mach number range from 0.5 to 1.4, and is controlled, via a feedback mechanism, by the air inlet main control valve. At a working section Mach number of 0.81 , and with a reservoir pressure of $800 \mathrm{kPa}$, the tunnel run-time is approximately $8 \mathrm{~s}$. Freestream velocity was monitored by a Pitot-static probe mounted in the working section and measured using a Furness Controls FCO14 micro manometer. A K-type thermocouple was used for the measurement of the freestream static temperature, which enabled temperature correction of the dynamic pressure. The nominal operating conditions are given in Table 1.

\begin{tabular}{lcccl}
\hline Quantity & Value & $\Delta_{\text {Dev }}$ & $\sigma_{\text {Dev }}$ & Units \\
\hline$M_{\infty}$ & 0.81 & 0.0027 & 0.0013 & - \\
$q_{\infty}$ & 29.8 & 0.1660 & 0.0799 & $\mathrm{kPa}$ \\
$p_{\infty}$ & 63.5 & 0.5566 & 0.2872 & $\mathrm{kPa}$ \\
$T_{\infty}$ & 259.4 & 5.3056 & 2.3482 & $\mathrm{~K}$ \\
\hline
\end{tabular}

Table 1: Nominal wind tunnel operating conditions. 


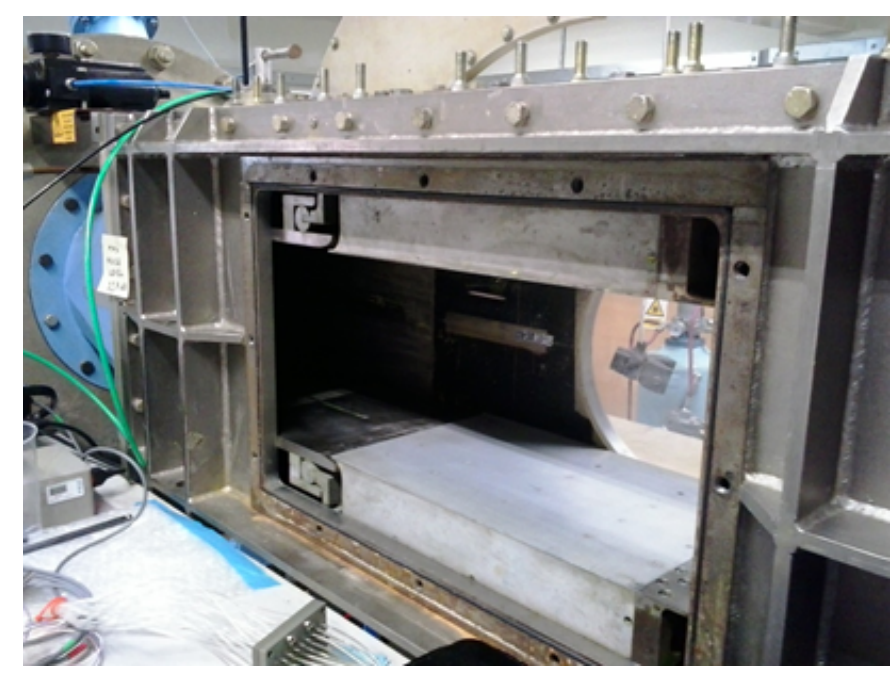

Figure 1: Transonic wind tunnel with side door removed; flow direction right to left.

Here the $\Delta_{\mathrm{Dev}}$ and $\sigma_{\mathrm{Dev}}$ columns indicate respectively the absolute maximum and the rms deviations from the reference value encountered in all the runs executed. The maximum deviation was $2 \%$ (though usually much less), while the repeatability of the results (i.e., the maximum deviation between any given pair of runs performed) was below $1 \%$, for pressure coefficient and OASPL, and less than $2 \%$ for the frequency spectra. The incoming boundary layer to the cavity was fully turbulent with a thickness of $8.8 \mathrm{~mm}$.

The cavity apparatus was built from a rectangular cut-out integrated into a tunnel wall insert such that the exit plane of the cavity was coincident with the tunnel side wall. The overall bay was $160 \mathrm{~mm}$ long, $32 \mathrm{~mm}$ wide and $32 \mathrm{~mm}$ deep, giving non-dimensional parameters, $L / D=5$ and $W / D=1$.

\subsection{Instrumentation and data acquisition}

A total of 27 pressure tappings were placed in the cavity floor. They were divided into three longitudinal rakes as shown in Figure 2. The longitudinal location of the pressure tappings was $x / L=0.1,0.2,0.3,0.4,0.5,0.6,0.7,0.8,0.9$ and at spanwise positions of $2 y / W=0 \pm 0.8125$. The analogue pressure signals where collected by a Scanivalve ZOC22B electronic pressure scanning module, capable of accepting 32 individual pressure sensors. Data was sampled at $20 \mathrm{kHz}$ for a total sample duration of 6.5536 seconds, or 131072 samples. Data was filtered during the acquisition phase to remove aliasing effects using a Bessel-type filter of 2nd order with a cut-off frequency of $10 \mathrm{kHz}$, a passband ripple of $\pm 1.5 \mathrm{~dB}$ at the cut frequency, and an attenuation slope of $40 \mathrm{~dB}$ per frequency decade.

\section{Data analysis}

The analysis of a signal containing only the power spectrum cannot provide evidence of any time-localized feature since only frequency information can be extracted. Furthermore, non-linear interactions will not be identified because of the suppression of all phase information. These problems can be overcome by expanding the signal post-processing techniques with time-frequency domain analysis methodologies and higher order spectral methods. The complementary methods of frequency-domain analysis and a time-frequency domain approach were used, therefore, for the purpose of data analysis. 


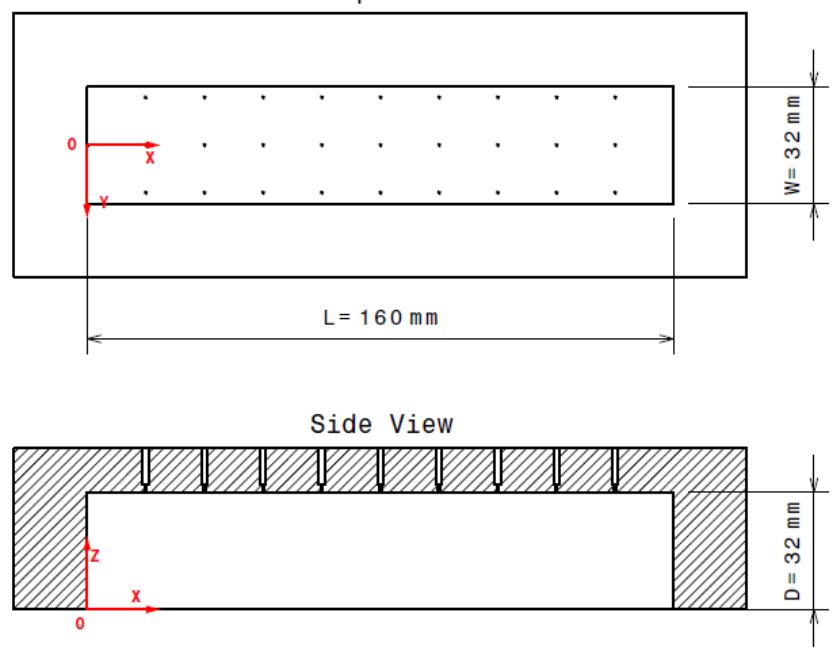

Figure 2: Cavity schematic and coordinate system.

\subsection{Frequency domain analysis}

Given a discrete-finite time series in the form $x_{n}$ with, $n=1,2, \ldots N$, sampled at a time step $\mathrm{d} t$, the discrete Fourier transform is defined as,

$$
\hat{X}_{k}=\sum_{j=1}^{N} x_{j} \cdot e^{-\frac{2 \pi k j i}{N}}
$$

Following this, the one-sided power spectral density (PSD) is defined as,

$$
\operatorname{PSD}(k)=\frac{2}{f_{s} \cdot N} \cdot \hat{X}_{k} \cdot \hat{X}_{k}^{*} \quad k=1,2 \ldots \frac{N}{2}+1
$$

The associated frequency vector is then defined as,

$$
f=(0,1,2 \ldots N / 2) \cdot \frac{f_{s}}{2 N}
$$

The common practice for a long time series is to divide it into a defined number of shorter overlapping elements, calculate the PSD for each segment, and then take the average. This is done to reduce the variance of the estimation. In this study, the analysed time series were divided into blocks with $50 \%$ overlap and with the number of samples equal to the ratio $f_{s} / f_{r}$, where $f_{s}$ is the sampling frequency and $f_{r}$ is the frequency resolution. A Hanning window was then applied to each block to reduce leakage effects. Finally, the PSD of the original signal was calculated by averaging the PSD of each block.

In order to discriminate between nonlinearly coupled waves and the spontaneously excited independent waves in a time series, it is necessary to adopt high-order (or non-linear) spectral analysis. Quadratic non-linearity implies that for a triad of frequencies, $f_{1}, f_{2}, f_{3}$, with corresponding phases $\phi_{1}, \phi_{2}, \phi_{3}$, the following equations are satisfied.

$$
\begin{gathered}
f_{1}+f_{2}=f_{3} \\
\phi_{1}+\phi_{2}=\phi_{3}+\vartheta
\end{gathered}
$$

Here $\vartheta$ is a constant phase angle. Equation 5 is the necessary condition for frequency coupling while Equation 6 is mandatory for phase coupling. More specifically, non-linearity is quantified using a quantity called bi-coherence (BC), which is a direct 
derivation from the bi-spectrum (BS), defined as the double Fourier transform of the third order moment, or more generally the third order cumulant.

$$
\operatorname{BS}\left(f_{1}, f_{2}\right)=\left\langle\hat{X}\left(f_{1}\right) \cdot \hat{X}\left(f_{2}\right) \cdot \hat{X}^{*}\left(f_{1}\right)\right\rangle
$$

If the bi-spectrum is normalised the bi-coherence spectrum (BC) is obtained as,

$$
\mathrm{BC}\left(f_{1}, f_{2}\right)=\sqrt{\frac{\left|\mathrm{BS}\left(f_{1}, f_{2}\right)\right|^{2}}{\left\langle\left.\hat{X}\left(f_{1}\right) \cdot \hat{X}\left(f_{2}\right)\right|^{2}\right\rangle \cdot\left\langle\left.\hat{X}\left(f_{3}\right)\right|^{2}\right\rangle}}
$$

The bi-coherence is bounded between 0 and 1 by the Schwarz inequality. According to Kim and Powers [8] the square of the bi-coherence, $\mathrm{BC}^{2}\left(f_{1}, f_{2}\right)$, can be interpreted as the fraction of power at $f_{3}$ due to the quadratic phase coupling between $f_{1}$, $f_{2}$, and $f_{3}$.

\subsection{Time-frequency domain analysis}

The Fourier transform is able to determine all the frequencies present in the signal, but it is not able to calculate the exact time at which a particular spectral component is present. Moreover, its accuracy relies on long time series (compared to the time scales of the problem) that are not always available. In addition, frequency domain analysis is valid only if the signal under consideration is statistically stationary. In many instances, however, this does not represent the reality of the situation, so it is preferable to analyse a signal in the time-frequency domain. The simplest technique is to use the short time Fourier transform (STFT). This technique is used to determine the sinusoidal frequency and phase content of local sections of a signal as it evolves in time. It is based on a procedure that divides the time series into shorter overlapping segments of equal length, considered statistically stationary, and to apply to each of them the Fourier transform in order to obtain the spectrum of each segment and hence a time-frequency picture (or map) of the signal. Letting $\widehat{S X}(k, n)$ be the value of the transform at a frequency, $k$, and time index, $n$, it is possible to write,

$$
\widehat{S X}(k, n)=\sum_{j=1}^{N} x_{j} \cdot w_{p}(j-n) \cdot e^{\frac{2 \pi k j i}{N}}
$$

Equation 9 is identical to Equation 2 apart from the term $w_{p}$, which is called the 'window of the transform' and is used to divide the original series into shorter segments of width $p$ (the derivation of the power spectral density is analogous to the Fourier transform, i.e. substituting $\widehat{X}_{k}$ with $\widehat{S X}$ in Equation 3). In this study, a Hanning window with $50 \%$ overlapping segments has been used. The characteristic of the STFT is that it is capable of identifying possible statistical non-stationary phenomena affecting the original time series. The apparent advantage of the STFT often disappears due to what is known as the problem of time-frequency resolution. The windowing in the STFT imposes a resolution in time (the width of the window) which implies a maximum available frequency resolution. This evolves into two antithetical situations. If high resolution in frequency is required, the resolution in time is sacrificed; if high resolution in time is required, the resolution in frequency is sacrificed. This problem is resolved by the wavelet transform, in which the dimension of the window varies for every spectral component, i.e. it changes when moving from low frequencies to higher ones. A wavelet is a function, real or complex, $\psi(\eta)$ with a zero mean localised in both time and frequency [9]. The wavelet transform can be both discrete (orthogonal basis) and continuous (non-orthogonal and orthogonal basis). In this study, a Morlet wavelet was used, consisting of a plane wave modulated by a Gaussian.

$$
\psi_{0}(\eta)=\pi^{-1 / 4} \cdot e^{i \omega_{0} \eta} \cdot e^{-\eta / 2}
$$


The Morlet is a complex, non-orthogonal wavelet basis with $\omega_{0}$ being the non-dimensional central frequency, posed equal to six, which is a standard value [9].

As stated by Torrence and Compo [10], the wavelet transform can be approximated by the inverse Fourier transform of the product of the DFT, of the time series $\widehat{X}_{k}$ (see Equation 2), and the complex conjugate of $\widehat{\psi}\left(s \omega_{k}\right)$, normalised in frequency space. The final equation then becomes,

$$
W(s, n)=\sum_{k=1}^{N} \hat{X}_{k} \cdot\left(\frac{2 \pi s}{\mathrm{~d} t}\right)^{1 / 2} \cdot \widehat{\psi}^{*}\left(s \omega_{k}\right) \cdot e^{i \cdot \omega_{k} n \mathrm{~d} t}
$$

The scales, $s$, of the Morlet wavelet transform, are correlated with the frequencies, in which the signal is decomposed, by the following equation,

$$
\frac{1}{f}=\frac{4 \pi}{\omega_{0}+\sqrt{2+\omega_{0}^{2}}} \cdot s
$$

For a non-orthogonal wavelet, such as the Morlet one, the set of scales to be used is discrete [9]. Once the transform has been calculated, it is possible to define the wavelet spectral power (WSP) as,

$$
\mathrm{WSP}(s, n)=10 \log _{10} \frac{W(s, n) \cdot W^{*}(s, n)}{\kappa^{2}} \cdot \frac{\mathrm{d} t}{s}
$$

Here $\kappa$ is a normalisation constant. In this study $\kappa$ was set equal to $2 \times 10^{-5} \mathrm{~Pa}$, the threshold of human hearing. From the wavelet transform, it is also possible to derive two additional quantities, the global wavelet spectral power (GWSP) and the wavelet average variance trend (WAVT). The global wavelet spectral power is defined as,

$$
\operatorname{GWSP}(s)=10 \log _{10} \frac{1 / N \cdot \sum_{n=1}^{N} W(s, n) \cdot W^{*}(s, n)}{\kappa^{2}} \cdot \frac{\mathrm{d} t}{s}
$$

This quantity is a smoothed version of the PSD obtained with the Fourier transform. The wavelet average variance trend is instead defined as,

$$
\operatorname{WAVT}(n)=10 \log _{10}\left(\frac{\mathrm{d} j \cdot \mathrm{d} t}{C_{\delta} \cdot \sigma^{2}} \cdot \sum_{j=0}^{j} \frac{W\left(s_{j}, n\right) \cdot W^{*}\left(s_{j}, n\right)}{s_{j}}\right)
$$

This quantity is a new time-series expressing the trend of the energy of the signal (and hence of the variance) with time. Expressing this variable in decibel units, once normalised by the variance of the original signal, will give a quantity for which positive values will express periods where the local signal power is above the signal's variance, while for negative values the opposite will be true.

The wavelet bi-spectrum, the wavelet bi-coherence, and the wavelet bi-phase can be defined in a similar way as for the bi-spectrum, the bi-coherence, and the bi-phase for the Fourier transform, and the interpretation is the same. The difference is that the former quantities can be defined over a sub-domain of the temporal length of the signal in order to analyse particular moments where statistically non-stationary behaviour is expected. The wavelet instantaneous bi-spectrum, using Equation 12 to change scale with frequency, is defined as:

$$
\operatorname{IWBS}\left(s_{1}, s_{2}, n\right)=W\left(s_{1}, n\right) \cdot W\left(s_{2}, n\right) \cdot W^{*}\left(s_{3}, n\right)
$$

where,

$$
\frac{1}{s_{3}}=\frac{1}{s_{1}}+\frac{1}{s_{2}}
$$


The instantaneous wavelet bi-coherence is finally expressed as,

$$
\operatorname{IWBC}\left(s_{1}, s_{2}, n\right)=\frac{\left|S\left(\operatorname{IWBS}\left(s_{1}, s_{2}, n\right) \cdot s_{s}^{-1}\right)\right|^{2}}{S\left(\left|W\left(s_{1}, n\right) \cdot W\left(s_{2}, n\right)\right|^{2} \cdot s_{s}^{-1}\right) \cdot S\left(\left|W\left(s_{3}, n\right)\right|^{2} \cdot s_{s}^{-1}\right)}
$$

Here $S\left(\right.$ ) is a smoothing operator in time and scale, i.e. $S(W(s, n))=S_{\text {scale }}\left(S_{\text {time }}(W(s, n))\right)$, as defined by Grinsted et al. [11]. For the Morlet wavelet, the combined operators are respectively,

$$
\begin{gathered}
\left.S_{\text {time }}(W(s, n))\right|_{s}=\left.\left(W(s, n) \cdot c_{1}^{\frac{-(n \mathrm{~d} t)^{2}}{2 s^{2}}}\right)\right|_{s} \\
\left.S_{\text {scale }}(W(s, n))\right|_{n}=\left.\left(W(s, n) \cdot c_{2} \Pi(0.2 s)\right)\right|_{n}
\end{gathered}
$$

Here $c_{1}$ and $c_{2}$ are normalisation constants determined numerically and $\Pi$ is the rectangular function. The variable $s_{s}$ is the non-dimensionalisation scale. Schulte [12] suggests that it is set equal to the maximum of $s_{1}$ and $s_{2}$ as this tended to reduce the over estimation of bi-coherence. In this study, however, due to the nature of the signals analysed, it was found that a good compromise was to set $s_{S}$ equal to $s_{3}$. As in the case of the bi-coherence, the wavelet bi-coherence is an indicator of quadratic coupling between the triad of waves defined by the scales $s_{1}, s_{2}$ and $s_{3}$ (see Equation 17). The interpretation of these new higher-order statistical quantities is the same as in frequency domain analysis. The difference is that, in this case, they are all based on a time-frequency domain transform of the signal, and hence they are able to capture any particular time-localised features.

In the analysis of waves, whose behaviour is not statistically stationary, it is useful to introduce a further quantity, called the 'index of persistence', or simply 'persistence' (PR). This value expresses the percentage of the signal duration in which a given frequency band is active, i.e. has enough power to be considered a feature of the flow and not simply just background noise. It is possible to write,

$$
\left.\mathrm{PR}\right|_{s_{1}} ^{s_{2}}=\sum_{n=1}^{N} \sum_{j=s_{1}}^{s_{2}} \frac{A(s, n)}{n j} \cdot 100
$$

Here $A(s, n)$ is the 'activity map', i.e. a map equal to 1 whenever the local wavelet power spectrum is above a reference value and 0 otherwise.

$$
A(s, n)= \begin{cases}1: & \frac{\mathrm{WPS}(s, n)}{\sigma^{2}} \geq \frac{1}{2} P_{s} \cdot \chi_{2}^{2}(p) \\ 0: & \frac{\mathrm{WPS}(s, n)}{\sigma^{2}}<\frac{1}{2} P_{s} \cdot \chi_{2}^{2}(p)\end{cases}
$$

Here $\chi_{2}^{2}$ is the chi-squared function with two degrees of freedom, $p$ is the desired confidence level, set equal to $95 \%$ in this study, and $P_{s}$ is a background red-noise spectrum, built using autocorrelation coefficients of the original time series. Equation 21 is then bounded between $0 \%$ (no activity) and $100 \%$ (constant activity) and is particularly useful to investigate how much a wave is active during the time-window analysed.

\subsection{Pressure data analysis}

When post-processing pressure signals, it is useful to define the sound pressure level (SPL) and the overall sound pressure level (OASPL), expressed in decibel units, calculated as follows,

$$
\begin{gathered}
\operatorname{SPL}(f)=10 \log _{10}\left(\frac{\operatorname{PSD}_{p_{f}}(f) \cdot \Delta f_{\mathrm{ref}}}{p_{\mathrm{ref}}^{2}}\right) \\
\text { OASPL }=20 \log _{10}\left(\frac{\sigma_{p}}{p_{\mathrm{ref}}}\right)
\end{gathered}
$$


Here $p_{\text {ref }}$ is the minimum audible pressure, equal to $2 \times 10^{-5} \mathrm{~Pa}$, which is defined as the threshold of human hearing, and $\Delta f_{\text {ref }}$ is a reference frequency set equal to $1 \mathrm{~Hz}$, which is used to make the new quantity non-dimensional (from Equation 3, PSD units are, for pressure signals, $\mathrm{Pa}^{2} / \mathrm{Hz}$ ).

When it is necessary to compare pressure signals obtained at different freestream conditions, it is convenient to use another non-dimensionalisation procedure, based on the freestream dynamic pressure instead of $p_{\text {reference }}$. Fluctuating pressure level (FPL) and overall fluctuating pressure level (OAFPL) are then defined as,

$$
\begin{gathered}
\mathrm{FPL}(f)=10 \log _{10}\left(\frac{\operatorname{PSD}_{p_{f}}(f) \cdot \Delta f_{\text {ref }}}{q_{\infty}^{2}}\right) \\
\text { OAFPL }=20 \log _{10}\left(\frac{\sigma_{p}}{q_{\infty}}\right)
\end{gathered}
$$

\subsection{Error estimation}

Since the data histories recorded had a finite nature, every value estimation was subjected to a level of uncertainty. To discern true features of the signal from uncorrelated noise various methodologies, according to the quantity under consideration, were used. For the PSD error estimation the procedure described by Newland [13] was used. Using $f_{r}=20 \mathrm{~Hz}$ a confidence interval on PSD of $-0.38 \mathrm{~dB}$ to $+0.39 \mathrm{~dB}$, relative to the calculated value, was obtained. The wavelet transform error was analysed using the procedure described by Torrence and Compo [10], that is the comparison of the obtained spectrum with a proper background red noise, constructed utilising a univariate lag-1 autoregressive process. Finally, for the bi-coherence uncertainty levels it was decided to use a Monte-Carlo method for the determination of proper confidence levels. The procedure involved the generation of 1000 surrogate datasets of noise that were then passed to the routine that calculated the quantity under consideration (bi-coherence, bi-phase etc.). From the obtained ensemble, the $P=100\left(1-\alpha_{p}\right)$ percentile was computed, where $P$ represented the threshold above which the bi-coherence value was considered a true feature and not rejected, and $\alpha_{p}$ is the statistical significance. The obtained value was then used as the threshold above which the feature under consideration was considered true. For all the above methodologies, a confidence level equal to $95 \%$ (or significance level equal to 0.05 ) was adopted.

\section{Results}

FPL and OAFPL plots of the current experiment were compared with data obtained by Henshaw [14], where a similar cavity, the M219 configuration, with $L / D=5$ was tested at a Mach number of 0.85 . Both sets of data are for the centreline rake. Although the two cases are broadly similar in their set-up, there are some significant differences between the two. The tests reported by Henshaw [14] were conducted at a larger scale giving a Reynolds number, based on cavity length, of approximately 9 million versus 2.2 million for the current study. Furthermore, the current study places the cavity in the sidewall of the wind tunnel and uses the natural turbulent boundary layer to generate an onset flow. Henshaw's tests were conducted with a sting-supported model embedded in a flat plate with a sharp leading edge. No measurements were made of the onset flow and hence the onset boundary layer profile is not known, or indeed if there was any separation of the boundary layer upstream of the cavity. Such experimental differences could explain the variation shown on the OAFPL data (Figure 3). The OAFPL for the current study assumed a W-shaped curve, with local minima at $x / L=0.2$ and $x / L=0.7$. Additionally, at all stations, the OAFPL values encountered were between 4.0 to 6.0 decibels higher than those recorded for the M219 cavity. 


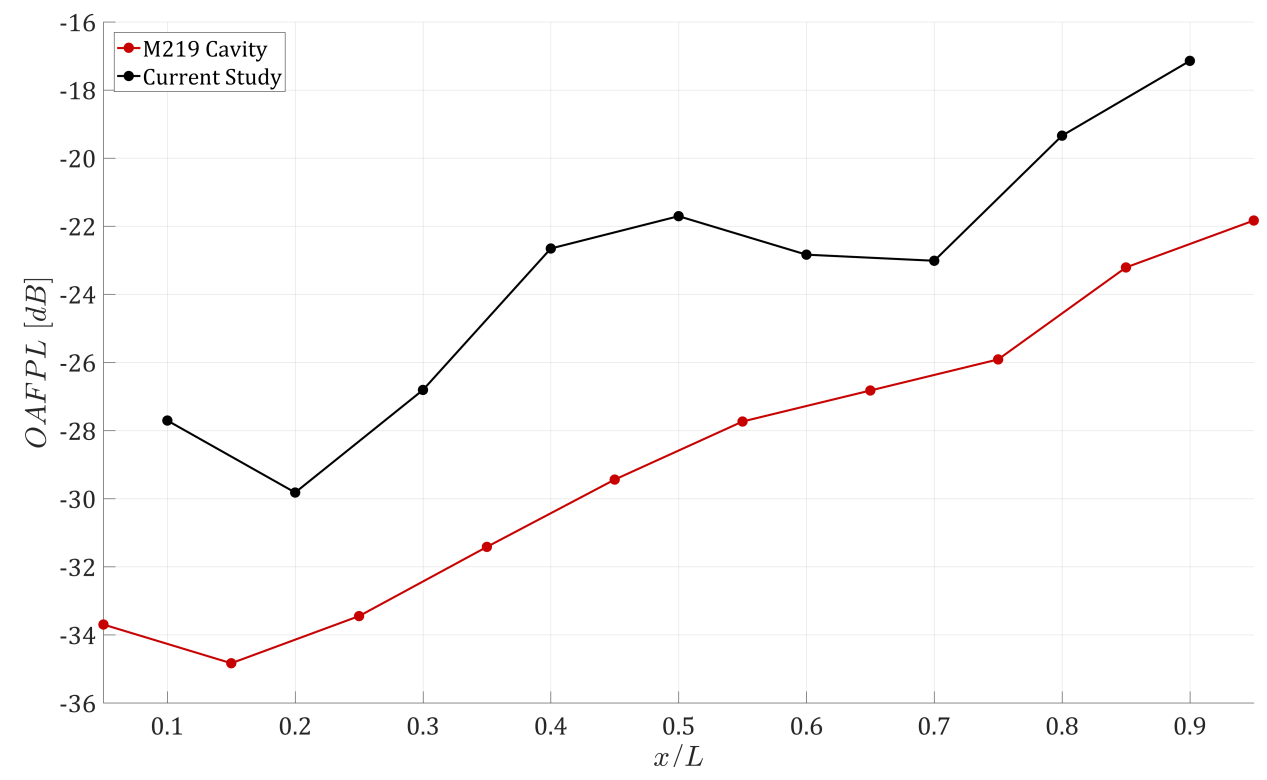

Figure 3: Comparison of the magnitude of OAFPL between the current study and the M219 cavity for the midspan rake [14].

The M219 cavity also had two local minima (at $x / L=0.15$ and $x / L=0.75$ ) although they were less pronounced than for the current study.

Differences were also observed in the FPL spectra. Here the rearmost stations were compared, as shown in Figure 4. Both cavities were characterised by the presence of three Rossiter-Heller tones, which differed in Strouhal number position and peak FPL values. Compared to the M219 data, the current study had higher peaks at the first two tones but lower at the third. Furthermore, all the M219 modes were located at lower Strouhal numbers, which were also lower than the predicted Rossier-Heller modes. It was possible to conclude that, while the compared cavities had similar geometries the different experimental details gave different acoustic responses. Despite the quantitative differences, when the spectra were compared, the overall qualitative trend was similar; a dominant second mode was accompanied by the first and third Rossiter-Heller tones.

Data analysis was divided into two phases. In Phase I, using a Fourier-based approach, Delprat's model [7] was verified and basic information regarding mode-switching, amplitude modulation, and phase coupling were extracted. In Phase II, using a wavelet-based approach, the analysis focused on obtaining a temporal picture of the non-linear interactions (AM/FM behaviours) proposed by Delprat's model. The purpose was to explain the frequency modulation, amplitude modulation, and the mode-switching behaviour, which usually characterise transonic cavity flows.

\subsection{Phase I: Fourier analysis approach}

From the analysis of the Fourier spectrum of the current study (Figure 5a) it was possible to identify three main modes, $M_{1}, M_{2}$ and $M_{3}$. All three tones were close to the theoretically-predicted values of the Rossiter-Heller equation, with $M_{2}$ being the dominant mode in the cavity. Following Delprat's decomposition, the carrier frequency, $M_{a}$, amplitude modulation frequency $M_{b}$, FM frequency, $M_{\Delta}$, and modulation parameter, $\gamma$, were calculated ${ }^{2}$. The data obtained are shown in Table 2. These relations indicated that the three main peaks could be identified as an amplitude modulation phenomenon of the

\footnotetext{
${ }^{2}$ This study reports frequency data as Strouhal number. To avoid confusion, $f_{a}, f_{b}$, and $\Delta f$ were renamed $M_{a}, M_{b}$ and $M_{\Delta}$.
} 


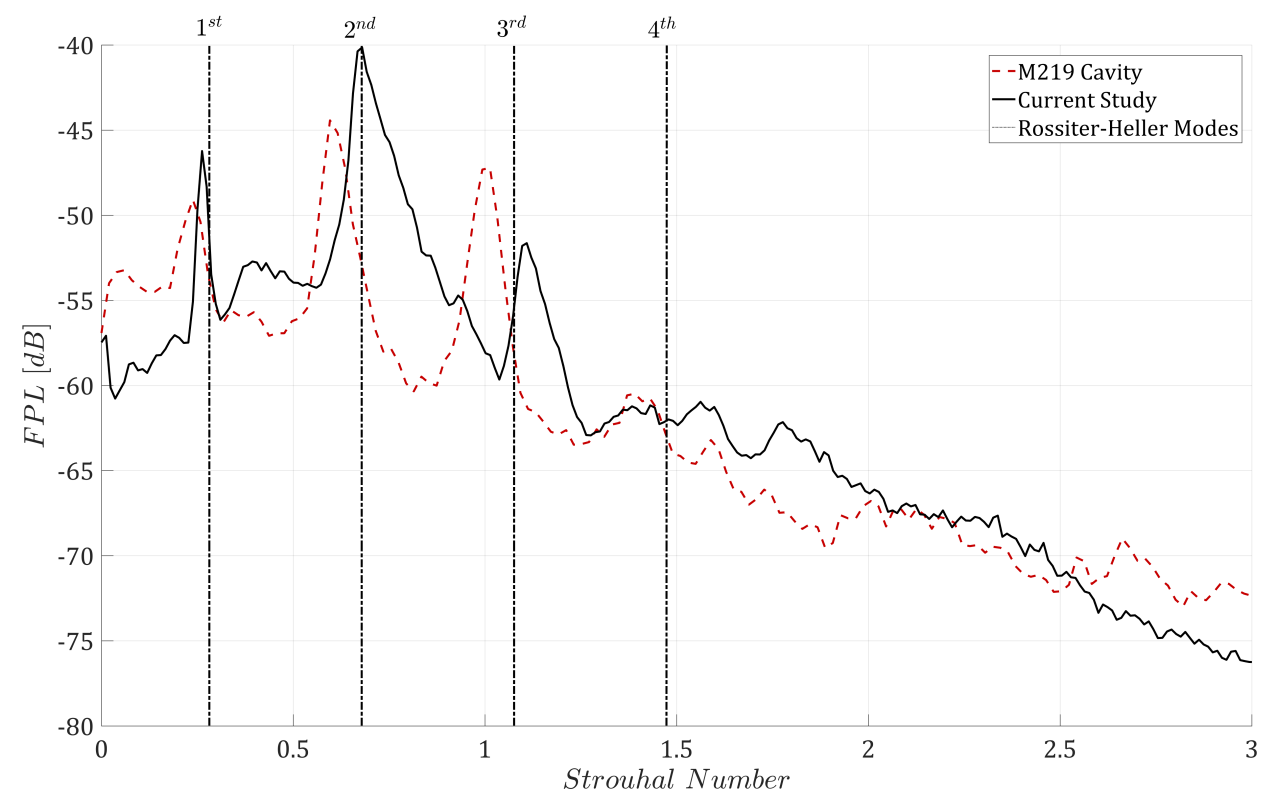

Figure 4: Comparison of the magnitude of the FPL between the current study $(x / L=0.9)$ and the M219 cavity $(x / L=0.95)[14]$ for the midspan rake.

\begin{tabular}{ccc}
\hline & Current Study & M219 [14] \\
\hline$M_{a}$ & 0.4218 & 0.4323 \\
$M_{b}$ & 0.1619 & 0.2188 \\
$M_{\Delta}$ & 0.0119 & 0.0596 \\
$\gamma$ & 0.384 & 0.506 \\
\hline
\end{tabular}

Table 2: Characteristic parameters derived for the current study and the M219 cavity [14] using Delprat's model [7].

fundamental resonant frequency $M_{a}$.

The same analysis was conducted for the M219 cavity data and again Delprat's theory was validated. Moreover, it was observed that whilst the two cavities had dissimilar spectral structures, their fundamental frequency was nearly the same. The difference was caused by the modulators in amplitude and frequency, which assumed values that were different from the current study. The difference is most likely due to the spectral response of the two cavities despite their similar geometry. It was then possible to calculate the predicted Rossiter-Heller tones as,

$$
\begin{aligned}
& M_{1}=M_{a}-M_{b} \\
& M_{2}=2 M_{a}-M_{b} \\
& M_{3}=3 M_{a}-M_{b}
\end{aligned}
$$

The predicted values are shown in Table 3 together with the measured values. The results show that the Rossiter tones can indeed be predicted with some degree of accuracy; better accuracy was achieved with the data from the current study.

As shown by Delprat [7], these relations indicated that the three main peaks, for both studies, could be identified as an amplitude modulation phenomenon of the fundamental resonant frequency, $M_{a}$. Although $M_{a}$ failed to appear in the spectrum as a defined peak it was possible to observe a small accumulation of energy in its corresponding frequency bin. It was also 

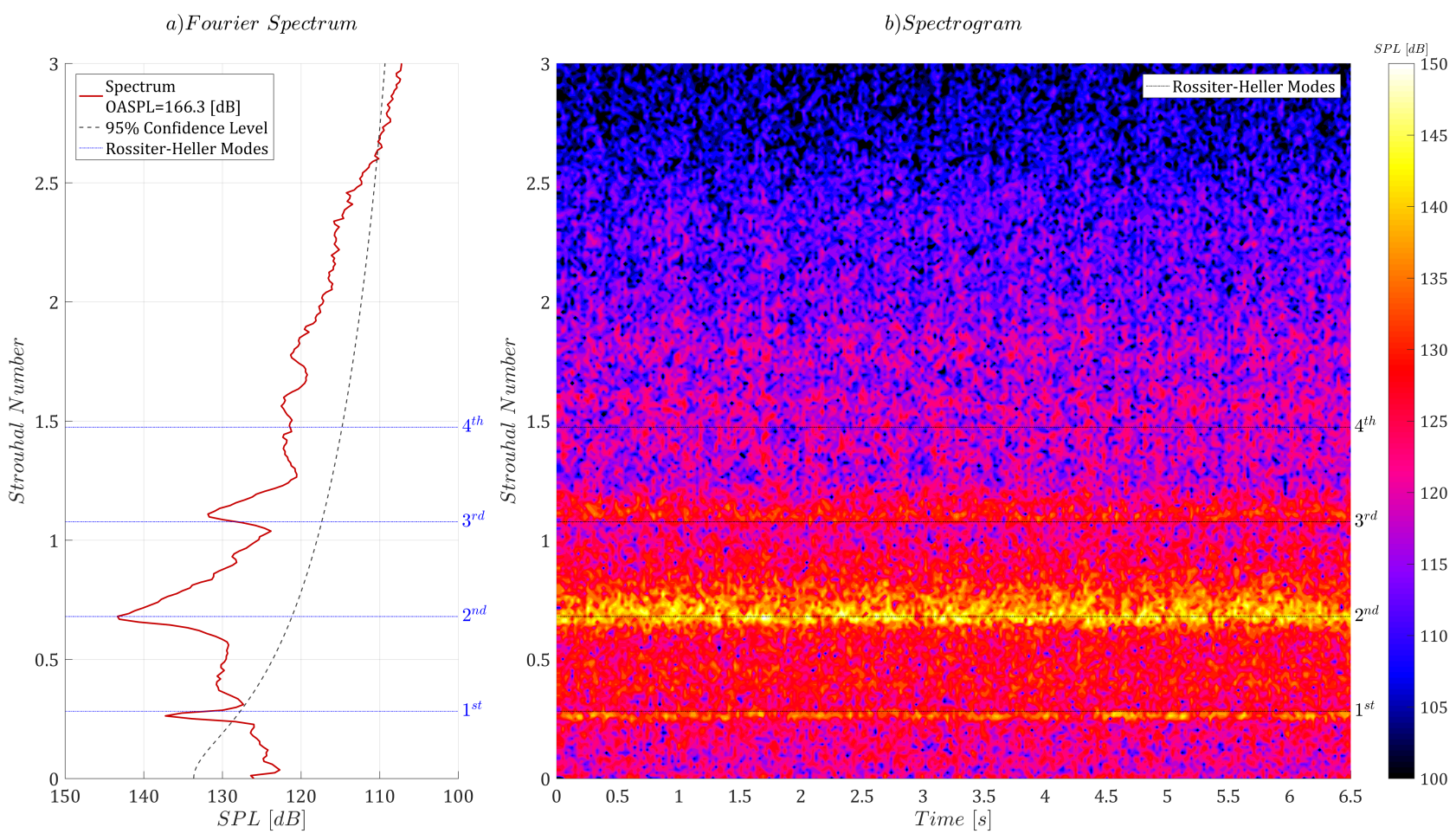

Figure 5: Frequency and time-frequency domain analysis based on the Fourier transform of the pressure signal for the current study $(x / L=0.9,2 y / D=0$, $z / D=1.0$ ); the frequency resolution is $20 \mathrm{~Hz}$.

\begin{tabular}{cccccccc}
\hline & \multicolumn{3}{c}{ Current Study } & & \multicolumn{3}{c}{ M219 [14] } \\
\cline { 2 - 3 } \cline { 6 - 8 } & Predicted [7] & Measured & FPL [dB] & & Predicted [7] & Measured & FPL [dB] \\
\hline$M_{1}$ & 0.2599 & 0.2624 & -46.24 & & 0.2135 & 0.2384 & -49.09 \\
$M_{2}$ & 0.6817 & 0.6768 & -40.15 & & 0.6458 & 0.5960 & -44.43 \\
$M_{3}$ & 1.104 & 1.106 & -51.64 & & 1.078 & 1.013 & -47.22 \\
\hline
\end{tabular}

Table 3: Characteristic parameters derived for the current study and the M219 cavity [14] using Delprat's model [7]. 
observed that whilst the two cavities had dissimilar spectral behaviour, their fundamental frequency was nearly the same. The difference was caused by the modulators in amplitude and frequency (most likely a consequence of the differences in the experimental setup), which assumed different values for each study, thereby causing differences in the spectral response.

Analysis of the time-frequency map (Figure 5b) indicated that all modes had an amplitude modulation behaviour, i.e. changed their power levels during the temporal evolution of the signal; this was particularly true for $M_{1}$. It was also possible to observe that the peaks corresponding to $M_{2}$ and $M_{3}$ had a wider base and side-peaks, features that did not appear in $M_{1}$. This indicating that for $M_{2}$ and $M_{3}$, as well as the amplitude modulation behaviour of Equations 28 and 29 (non-linear coupling between the harmonics of $M_{a}$ and $M_{b}$ ) were also affected by a frequency modulation corresponding to $\mathrm{M}_{\Delta}$. In fact for the current study, the mean value of the difference in Strouhal number between the side-band peaks of $M_{2}$ and $M_{3}$ was calculated to be 0.0224 , which is approximately equal to $2 M_{\Delta}(0.0238)$, again confirming Delprat's model validity.

From the analysis of the pressure signal, with higher order spectral methods (the Fourier-based bi-coherence given by Equation 8), and as predicted by Delprat's hypothesis [7], it was not possible to observe the interaction of the carrier and the modulator frequencies, which defined the system. Only two relevant couplings were observed (Figure 6), with BC values of 0.40 . These were formed, respectively, by the triads $\left(M_{2}, M_{2}, 2 M_{2}\right)$ and $\left(M_{3}, M_{2}, M_{3}+M_{2}\right)$. Nevertheless, this still left open the question as to whether $M_{a}$ interacted with some Rossiter-Heller tones to reinforce higher and lower ones, or if the observed couplings indirectly represented the non-linear interaction of $M_{a}$ and $M_{b}$. Since, as shown in the STFT analysis (Figure 5), all Rossiter-Heller tones exhibited amplitude modulation behaviour and since, during the temporal evolution of the signal, the location of a mode's peak varied along the Strouhal axis, the natural consequence was to also expect an intermittency in the non-linear interactions. It was decided, therefore, that in order to address these questions the signal would be analysed using the wavelet transform. This post-processing approach not only enabled observation of the temporal evolution (and hence the intermittency) of the non-linear interactions, but also enabled zooming along the time axis without losing frequency resolution.

\subsection{Phase II: wavelet analysis approach}

The temporal duration of the signal was many orders of magnitude larger than the natural scales of the problem. As discussed in the previous paragraph the biggest time scale of the problem (the lowest frequency) was $M_{\Delta}$, corresponding to $0.0513 \mathrm{~s}$. Comparing this value with the signal temporal length $(6.5536 \mathrm{~s})$, the two values have a ratio of the order of 1:100. This was an adequate number from a statistical point of view, nevertheless, it introduced difficulties when it was zooming the time-frequency map along the time scale in order to obtain a better view of the temporal evolution of the modes. The natural consequence was to extract sub-intervals of the original signal and this operation was performed with the following criteria. From the STFT map it the position of the dominant mode during the temporal evolution of the signal (see Figure 7) was extracted. This value indicated, at each time bin, the Strouhal number corresponding to the highest value of the SPL spectrum. The analysis shown in Figure 7 confirmed that for most of the time the highest mode in the spectrum was the second Rossiter-Heller tone $\left(M_{2}\right)$ with occasional occurrences of $M_{1}$ being the dominant tone. It was then decided to extract two time intervals with opposite characteristics. The first one, labelled $\Delta T_{1}$, which was between $2.4 \mathrm{~s}$ and $2.5 \mathrm{~s}$, indicated a period of the signal in which the dominant mode did not vary. The second time interval, labelled $\Delta T_{2}$, covering the interval from $3.5 \mathrm{~s}$ to $3.6 \mathrm{~s}$, represented a period in which a mode switch between the first and second Rossiter-Heller tones occurred. The $0.1 \mathrm{~s}$ intervals had a duration of at least twice the largest time-scale of the problem.

Wavelet analysis of the two time intervals are shown in Figures 8 and 9. Figure 8 shows clearly the dominance of the 


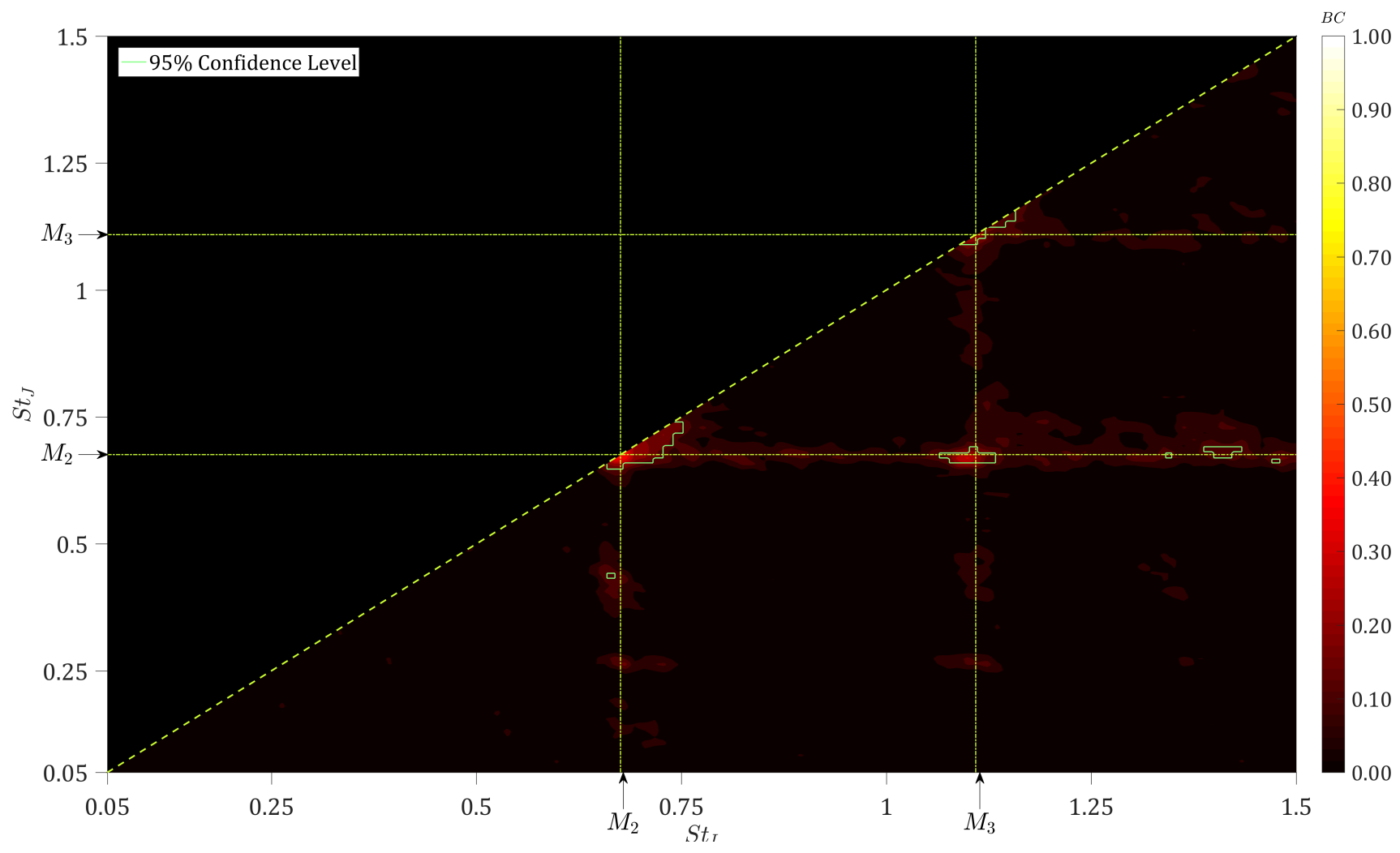

Figure 6: Bi-coherence analysis of pressure signal for the current study $(x / L=0.9,2 y / D=0, z / D=1.0)$; the frequency resolution is $20 \mathrm{~Hz}$. This figure represents all the possible combinations of the frequency vector, spanning from 0.0 to $\mathrm{St}_{n}$. The coordinates of each point in the ( $\left.\mathrm{St}_{i}, \mathrm{St}_{j}\right) \mathrm{plane}$ form, with their algebraic sum, a triad of frequencies whose squared bi-coherence value is expressed at that precise location. The symmetrical nature of the transform properties [8] enable it to be applied only inside the triangle defined by the coordinates $\left[0,0 ; \mathrm{St}_{n / 2}, \mathrm{St}_{n / 2} ; \mathrm{St}_{n}, 0\right]$ (note that this particular case shows a zoom on the left side of the validity domain for ease of visualization). The squared bi-coherence varies from 0.0 to 1.0 whereby 0.0 denotes a complete absence of coupling while 1.0 indicates that the three frequencies are $100 \%$ power coupled, i.e. the power content of one of them is induced by the non-linear interaction of the other two. Since the energy transfer process has no sign in the supporting mathematical formula, there is no way of telling whether the energy is passed through a sum $\left(\mathrm{St}_{k}=\mathrm{St}_{i}+\mathrm{St}_{j}\right)$ or difference $\left(\mathrm{St}_{i}=\mathrm{St}_{k}-\mathrm{St}_{j}\right.$ or $\left.\mathrm{St}_{j}=\mathrm{St}_{k}-\mathrm{St}_{i}\right)$ type process. Thus, the sum type process shown in the figure does not imply anything regarding the direction of the energy flow. 


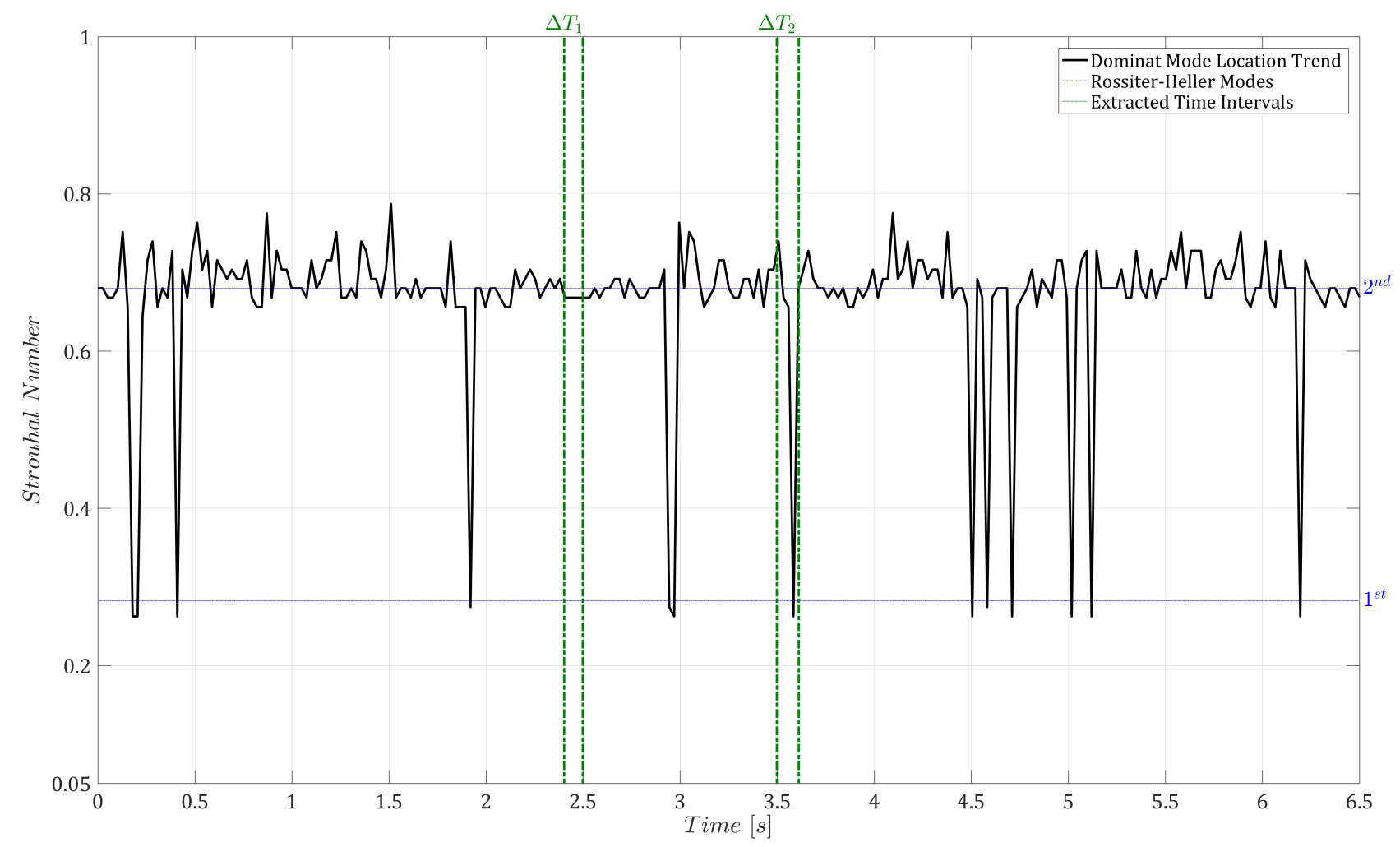

Figure 7: Identification of the dominant modes during the time evolution of pressure signals at $x / L=0.9,2 y / D=0, z / D=1.0$. Data was obtained from the time-frequency map of Figure 5. The vertical bands, labelled $\Delta T_{1}$ and $\Delta T_{2}$, indicate the intervals processed with the wavelet transform.

second Rossiter-Heller tone. The first mode $\left(M_{1}\right)$ was observed to have a strong intermittency, i.e. a small burst of activity separated by long periods of inactivity. Additionally, the allocated power associated with $M_{1}$ was an order of magnitude lower than $M_{2}$. No clearly identifiable trace was observed for the third mode, which had a trend similar to general noise, although occasional periods of activity did appear. The calculated PR indices for interval $\Delta T_{1}$ were $37.3 \%$ for $M_{1}, 81.3 \%$ for $M_{2}$, and $15.9 \%$ for $M_{3}$. Such values confirmed the intermittency nature of the Rossiter-Heller tones, especially $M_{1}$ and $M_{3}$, which were not active for the major part of the time interval.

The situation in the second time interval, $\Delta T_{2}$, was different. Figure 9, whilst still indicating $M_{2}$ as the dominant mode, revealed an increased activity of $M_{1}$, which explained the observed mode switch during $\Delta T_{2}$ in Figure 7 . The mode switch occurred between $3.58 \mathrm{~s}$ and $3.59 \mathrm{~s}$, during which $M_{1}$ became the dominant mode and $M_{2}$ disappeared. The PR indices of $M_{1}$, $M_{2}$, and $M_{3}$ were $51.91 \%, 76.40 \%$, and $21.87 \%$ respectively. Compared to the values observed in the interval $\Delta T_{1}, M_{1}$ and $M_{3}$ increased their value of PR, while $M_{2}$ decreased; this suggests a re-arrangement of the energy distribution between the modes.

To obtain a more accurate view of the signal's structure HOSA methodologies, based on the wavelet transform, were used. The utilisation of the instantaneous wavelet bi-coherence (Equation 18) enabled the identification of all quadratic interactions present in the signal during its temporal evolution. Delprat [7] highlighted the inability of the bi-spectral analysis to identify the non-linear interactions of $M_{a}$ and $M_{b}$ in the Rossiter-Heller tones. Her assumption relied on the fact that it was impossible to observe a non-linear interaction between two modes that did not appear in the spectrum. Nevertheless, considering Equations 28 and 29, are originated $M_{2}$ and $M_{3}$ through the appearance of the harmonics of $M_{a}$ and such passage of energy can indeed 


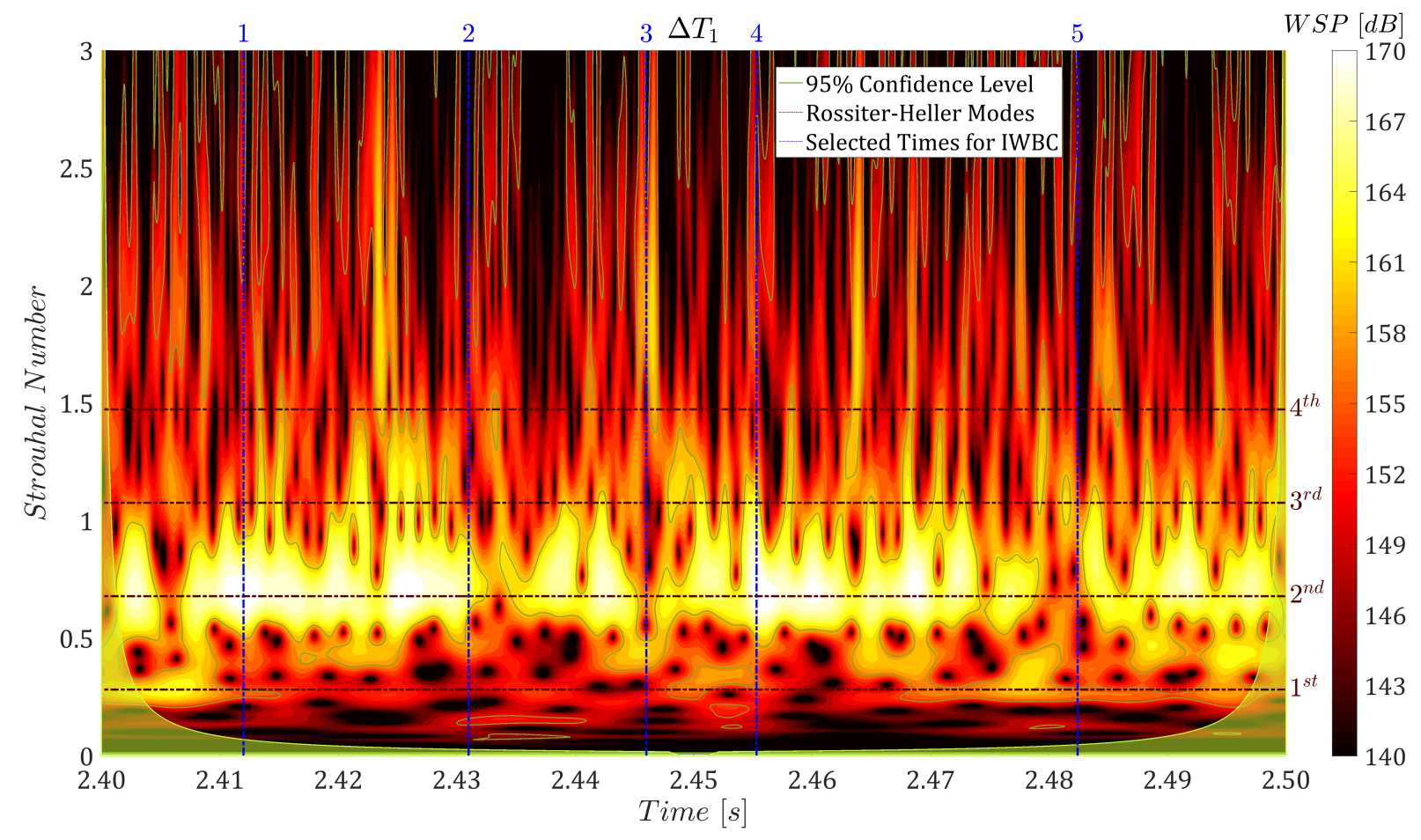

Figure 8: Wavelet spectral power of the pressure signals at $x / L=0.9,2 y / D=0, z / D=1.0$ (time interval $\Delta T_{1}$ ). The map color reflects, at each point, the WSP allocated at a particular time and Strouhal number. Confidence-level thresholds are drawn as green contour lines. The map also shows the cone-of-influence areas (shaded green at the edges), which regroups all the points influenced by the finite nature of the signal (also referred to as the signal truncation transform error).

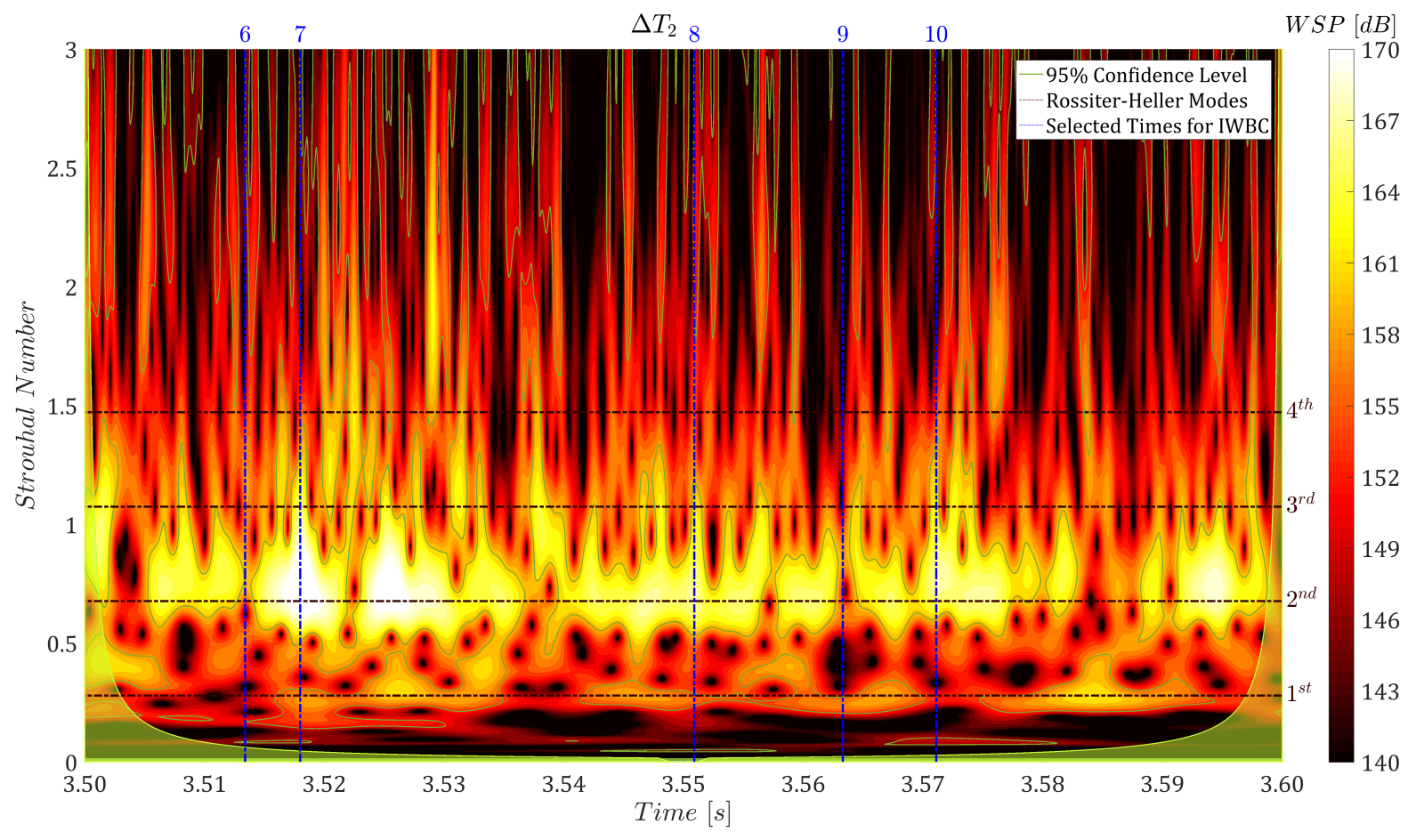

Figure 9: Wavelet spectral power of the pressure signals at $x / L=0.9,2 y / D=0, z / D=1.0\left(\right.$ time interval $\left.\Delta T_{2}\right)$. 

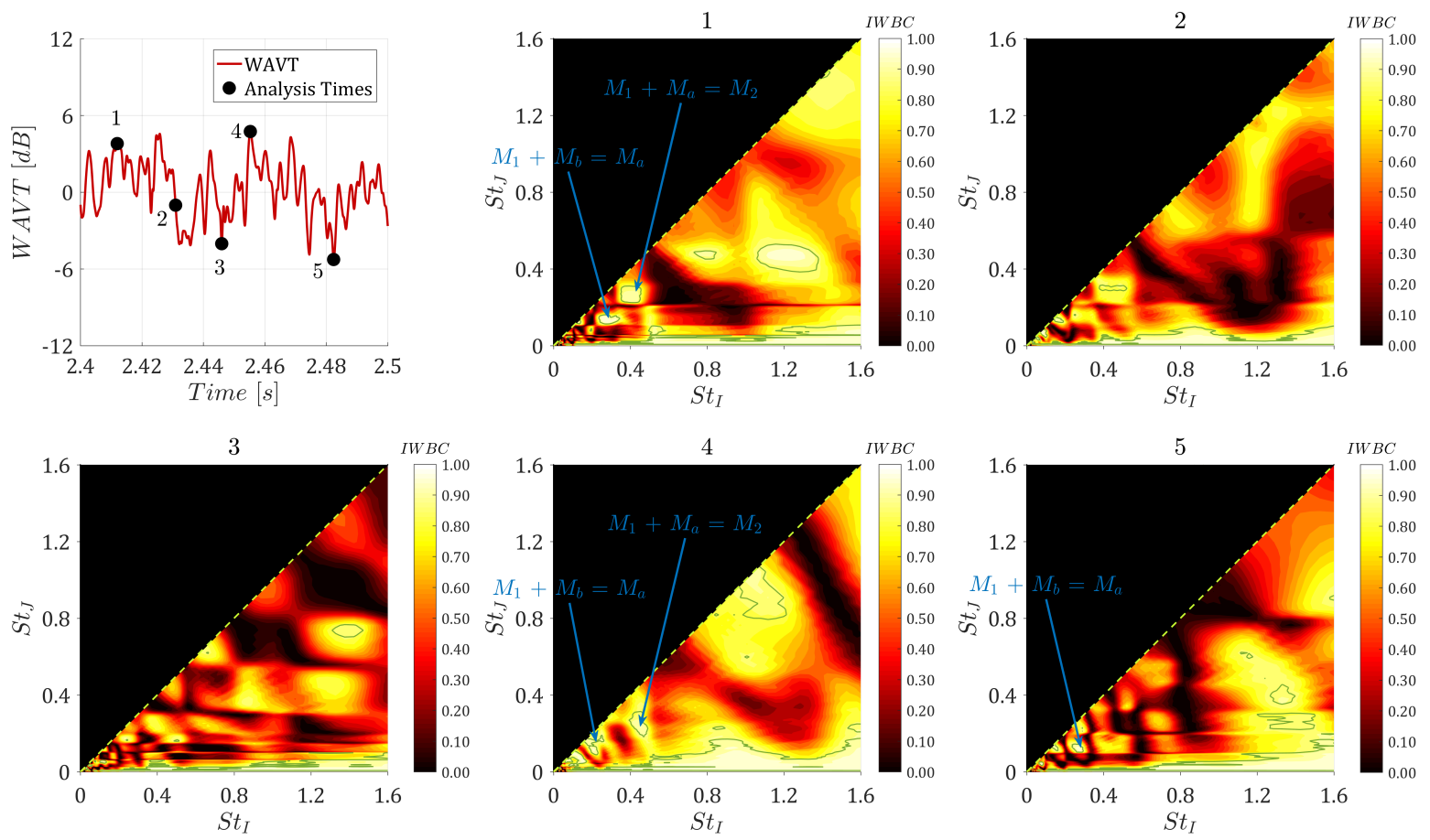

Figure 10: IWBC analysis of time period $\Delta T_{1}$. Each IWBC plot (1-5) corresponds to the intervals identified in the accompanying WAVT time history. Green contours represent the $95 \%$ confidence level boundaries.

be unveiled by a time-frequency analysis such is the IWBC.

For the time intervals $\Delta T_{1}$ and $\Delta T_{2}$ a total of $2001 \mathrm{bi}$-coherence maps were generated. Attention was then focussed on those maps in which mode switching or strong power output variations were observed. A total of ten time samples were selected, with samples 1 to 5 corresponding to $\Delta T_{1}$ and samples 6 to 10 corresponding to $\Delta T_{2}$. The time samples were chosen to represent particular characteristics of the flow such as local minima or maxima of the WAVT curve, instances in which no Rossiter-Heller mode was present, and switching of the dominant mode between $M_{1}$ and $M_{2}$. The results are reported in Figure 10 for $\Delta T_{1}$ and Figure 11 for $\Delta T_{2}$. Both figures are accompanied by the WAVT trend of the signal, expressing the instantaneous power output of the pressure fluctuations divided by the averaged power output of the signal. Inspection of the IWBC maps resulted in the determination of particular frequency combinations with high bi-coherence $(>0.95)$ indicating an exchange of significant energy, via quadratic nonlinearities, between the modes involved.

A common feature observed in both time intervals was that, no matter what time sample was selected, the lower bound of the IWBC maps (close to the origin of the $\mathrm{St}_{J}$ axis) showed bi-coherence values of 1.0. This area, which reflected the interaction of $M_{\Delta}$ with all other modes present in the spectrum, indicated a strong correlation between the lowest modulating frequency and all the other characteristic modes induced by shear layer fluctuations. As pointed out by Basley et al. [15] this was the influence of the centrifugal instabilities along the recirculating flow inside the cavity. The fact that the IWBC indicated that the low Strouhal number interaction persisted for the whole temporal evolution of the signal is important because, even if in particular time samples the main Rossiter-Heller modes were not active, the instabilities persisted and continued to exchange energy across a wideband spectrum. Thus, the intrinsic unsteadiness of the recirculating flow was a mechanism not originated by the instabilities of the shear layer, but by the fact that the domain was three-dimensional and oscillations originated in the 

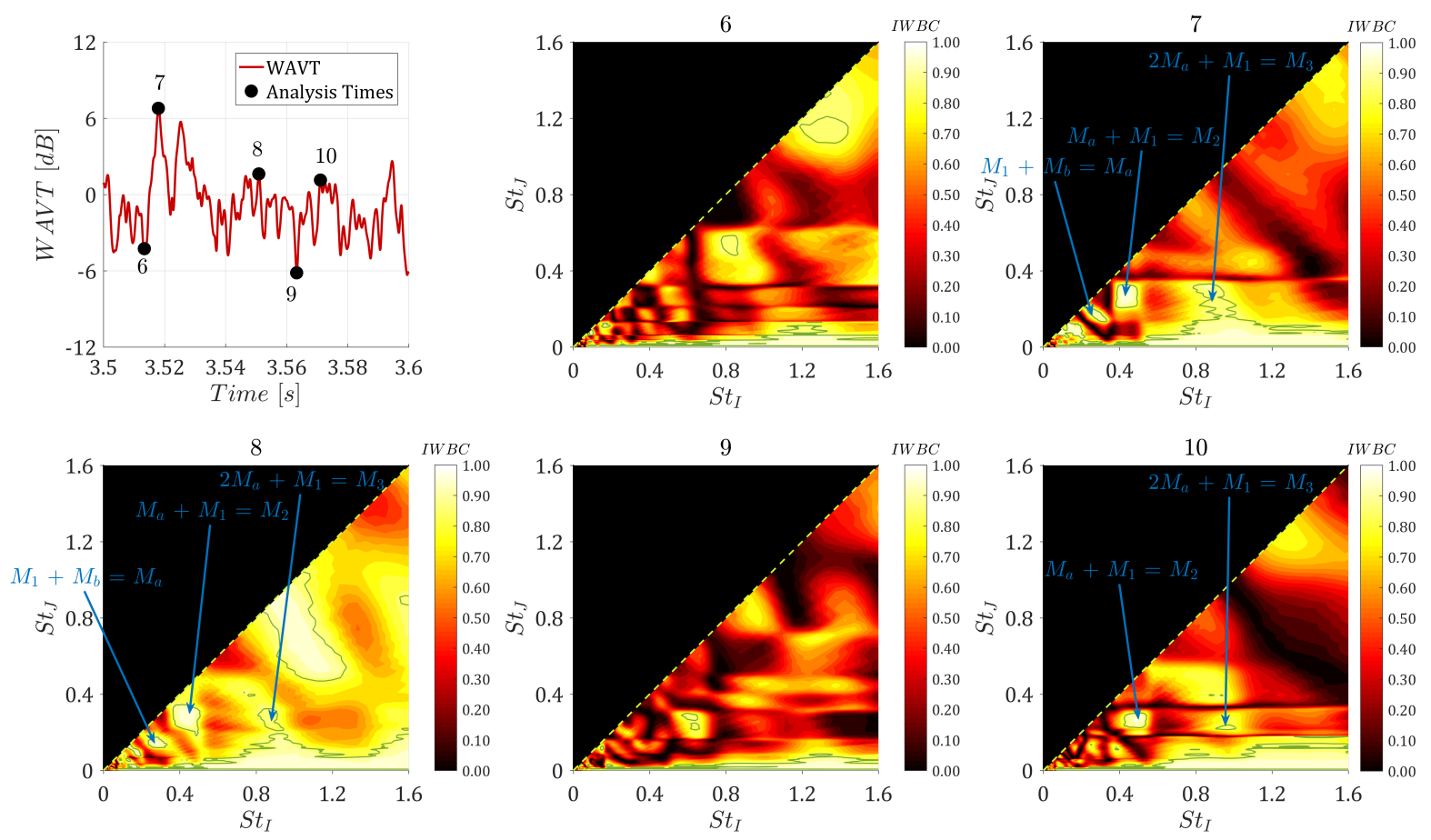

Figure 11: IWBC analysis of time period $\Delta T_{2}$. Each IWBC plot (6-10) corresponds to the intervals identified in the accompanying WAVT time history. Green contours represent the $95 \%$ confidence level boundaries.

transverse dimension of the cavity as well.

The five selected time samples (labelled 1-5 in Figure 10) of $\Delta T_{1}$ represented particular characteristics. Time samples with identification numbers 1 and 4 represented two conditions where the signal assumed a local maximum in the WAVT curve and $M_{2}$ was the dominant tone (indicated by the blue bands in Figure 8). Number 5 reflected a condition where the WAVT assumed a local minima and $M_{1}$ was the dominant mode. Finally, numbers 2 and 3 were characterised by a WAVT value below the reference (i.e. less than $0 \mathrm{~dB}$ ) and conditions on the wavelet map where the signal underwent a mode-off phase, with the main Rossiter-Heller modes disappearing for a brief period. The observed noteworthy couplings for each time sample are reported in Table 4.

In time samples 1 and 4 the combinations $M_{1}+M_{b}=M_{a}$ and $M_{1}+M_{a}=M_{2}$ (which are essentially a re-arrangement of Equations 27 and 29) were observed. Since the bi-coherence for these combinations was 1.0 it was possible to confirm the existance of a coupling between $M_{a}$ and $M_{b}$, and the Rossiter-Heller tones $M_{1}$ and $M_{2}$, confirming that the latter was originated by the interaction of the former. By comparison, in time samples 2 and 3, the WAVT of the signal assumed a local minima and/or values below $0 \mathrm{~dB}$, i.e. the signal was in a period of low-energy levels, in which the power emitted by the pressure oscillations was weaker respect to the average power. Here the wavelet analysis indicated the absence of any Rossiter-Heller mode and, in the associated IWBC map, no particular couplings were observed. The situation for time sample 5 was different, albeit corresponding to a local WAVT minima. The combination $M_{1}+M_{b}=M_{a}$ was present, correlated by the fact that $M_{1}$, in this temporal frame, was the dominant mode, while $M_{2}$ was inactive.

In the case of $\Delta T_{2}$ the selection of the time samples followed a similar logic to $\Delta T_{1}$ and the observed combinations are reported in Table 5. Time samples 6 and 9 corresponded to local WAVT minima (see Figure 11) and the absence of activity of 


\begin{tabular}{ccc}
\hline Time id & Signal time $[\mathrm{s}]$ & \multicolumn{1}{c}{ Mode couplings } \\
\hline 1 & 2.4120 & $\begin{array}{c}M_{1}+M_{b}=M_{a} \equiv M_{1}=M_{a}-M_{b} \\
M_{1}+M_{a}=M_{2} \equiv M_{2}=2 M_{a}-M_{b}\end{array}$ \\
\cline { 3 - 3 } 2 & 2.4310 & None \\
3 & 2.4460 & None \\
\cline { 3 - 3 } & & \multicolumn{2}{c}{$M_{1}+M_{b}=M_{a} \equiv M_{1}=M_{a}-M_{b}$} \\
4 & 2.4553 & $M_{1}+M_{a}=M_{2} \equiv M_{2}=2 M_{a}-M_{b}$ \\
\cline { 3 - 3 } 5 & 2.4824 & $M_{1}+M_{b}=M_{a} \equiv M_{1}=M_{a}-M_{b}$ \\
\hline
\end{tabular}

Table 4: Notable mode couplings extracted from the analysis of Figure 10 (time interval $\Delta T_{1}$ ).

any Rossiter-Heller tones in the wavelet map (the blue bands in Figure 9). Hence, no particular couplings were observed in the IWBC maps. In contrast samples 7 and 8, corresponded to local WAVT maxima with all three Rossiter-Heller tones present in the wavelet time-frequency spectrum. The couplings observed, all with bi-coherence values equal to unity, were the already encountered $M_{1}+M_{b}=M_{a}$ and $M_{1}+M_{a}=M_{2}$ (rearrangements of Equations 27 and 28), and the new $M_{1}+2 M_{a}=M_{3}$ (rearrangement of Equation 29). The last time-sample analysed, number 10, corresponded to a local WAVT maximum. In this case, however, as shown in the wavelet map, only $M_{2}$ and $M_{3}$ were active and, in accordance with the IWBC analysis, resulted in the occurrence of the $M_{1}+M_{a}=M_{2}$ and $M_{1}+2 M_{a}=M_{3}$.

The IWBC transform was demonstrated, therefore, to be capable of identifying the generation of the Rossiter-Heller tones as the result of strong energy exchange between a carrier frequency $M_{a}$ (and its relative harmonics) and a modulating frequency $M_{b}$. It was also observed that, whilst $M_{1}$ could also be active in low energy conditions, i.e. with WAVT values below $0 \mathrm{~dB}$, $M_{2}$ and $M_{3}$ were present only when the WAVT curve had values greater than $0 \mathrm{~dB}$. This implied that the generation of the harmonics of $M_{a}$ required stronger fluctuations in the shear layer, i.e. more energy produced by the resonant mechanism. Nevertheless, as wavelet analysis showed, the naturally-occurring condition in the signal spectrum was for the presence of $M_{2}$ flanked by weaker $M_{1}$ and $M_{3}$, with the latter being sporadically active during the temporal evolution of the signal. This indicated that, while the interaction between $M_{a}$ and $M_{b}$ can occur at low energy states, the tendency of the flow was to stabilise towards higher energy levels, such as the predominance of $M_{2}$ (and hence of the first harmonics of $M_{a}$ ) in the spectrum.

\section{Conclusions}

Transonic wind tunnel experiments were conducted on a rectangular cavity of length-to-depth ratio equal to five at a Mach number of 0.81. Pressure readings at ports located in the cavity's floor were processed using non-linear spectral analysis techniques. The analysis of the spectrum indicated that the resonant peaks were the result of the interaction of a carrier mode $M_{a}$ (and its harmonics) and a modulating frequency $M_{b}$. Additionally, it was observed that all modes underwent a further modulation mechanism induced by $M_{\Delta}$, the lowest peak appearing in the spectrum. These findings validated the model proposed by Delprat [7] and similar results were obtained when the results were compared with the M219 cavity at similar operating conditions. 


\begin{tabular}{|c|c|c|}
\hline Time id & Signal time [s] & Mode couplings \\
\hline 6 & 3.5134 & None \\
\hline 7 & 3.5180 & $\begin{array}{c}M_{1}+M_{b}=M_{a} \equiv M_{1}=M_{a}-M_{b} \\
M_{1}+M_{a}=M_{2} \equiv M_{2}=2 M_{a}-M_{b} \\
M_{1}+2 M_{a}=M_{3} \equiv M_{3}=3 M_{a}-M_{b}\end{array}$ \\
\hline 8 & 3.5509 & $\begin{array}{c}M_{1}+M_{b}=M_{a} \equiv M_{1}=M_{a}-M_{b} \\
M_{1}+M_{a}=M_{2} \equiv M_{2}=2 M_{a}-M_{b} \\
M_{1}+2 M_{a}=M_{3} \equiv M_{3}=3 M_{a}-M_{b}\end{array}$ \\
\hline 9 & 3.5633 & None \\
\hline 10 & 3.5711 & $\begin{array}{l}M_{1}+M_{b}=M_{a} \equiv M_{1}=M_{a}-M_{b} \\
M_{1}+M_{a}=M_{2} \equiv M_{2}=2 M_{a}-M_{b}\end{array}$ \\
\hline
\end{tabular}

Table 5: Notable mode couplings extracted from the analysis of Figure 11 (time interval $\Delta T_{2}$ ).

Comparison of the current study with the M219 cavity model showed that $M_{b}$ had a different magnitude, even if the two experiments had comparable Mach number and length-to-depth ratios. $M_{a}$, however, was similar for both cavities. This could suggest that while $M_{a}$ is dependent on a general cavity parameter such as the $L / D$ ratio or Mach number, $M_{b}$ might be the result of other factors more connected to local details of the geometry.

High order spectral analysis, based on the instantaneous wavelet bi-coherence (IWBC) method, was able to identify, at individual samples in the pressure-time signal, that the interaction between $M_{a}$ and $M_{b}$ was responsible for the creation of the Rossiter-Heller tones. The IWBC technique was also capable to correlate the mode switching phenomenon, as well as the deactivation of the resonant tones during the temporal evolution of the signal, to changes in the coupling of the fundamental tones $M_{a}$ and $M_{b}$.

Finally, it was observed that for the harmonics of $M_{a}$ to exist (and hence for $M_{2}$ and $M_{3}$ to be active in the spectrum) it was fundamental that the signal wavelet average variance trend had values greater than $0 \mathrm{~dB}$, that is, the power produced by the shear layer instabilities had to be above the average power expressed by the signal.

\section{Acknowledgements}

The authors would like to thank Dr Mark Finnis for his help with the wind tunnel experiments as well as his suggestions regarding the wavelet analysis.

\section{References}

[1] D. Rockwell and E. Naudasher. Review - self sustaining oscillations of flow past cavities. J. Fluid Eng., 100(2): $152-165,1978$. 
[2] J. E. Rossiter. Wind-tunnel experiments on the flow over rectangular cavities at subsonic and transonic speeds. Reports and Memoranda 3438, Aeronautical Research Council, 1966.

[3] H. Heller, D. Holmes, and E. Covert. Flow-induced pressure oscillations in shallow cavities. J. Sound Vib., 18:545-553, 1971.

[4] M. B. Tracy and E. B. Plentovich. Characterization of cavity flow fields using pressure data obtained in the Langley 0.3-meter transonic cryogenic tunnel. Tech. Rep. TM-4436, NASA Langley Research Center, Hampton, VA, USA, March 1993.

[5] K. K. Ahuja and J. Mendoza. Effects of cavity dimensions, boundary layer, and temperature on cavity noise with emphasis on benchmark data to validate computational aeroacoustic codes. Tech. Rep. CR-4653, NASA Langley Research Center, Hampton, VA, USA, 1995.

[6] M. A. Kegerise, E. F. Spina, S. Garg, and L. N. Cattafesta. Mode-switching and nonlinear effects in compressible flow over a cavity. Phys. Fluids, 16(3):678-687, 2004.

[7] N. Delprat. Low-frequency components and modulation processes in compressible cavity flows. J. Sound Vib., 329(22): 4797-4809, 2010.

[8] Y. C. Kim and E. J. Powers. Digital bispectral analysis and its applications to nonlinear wave interactions. IEEE T. Plasma Sci., 7(2):120-131, 1979.

[9] M. Farge. Wavelet transforms and their application to turbulence. Annu. Rev. Fluid Mech., pages 355-457, 1992.

[10] C. Torrence and G. P. Compo. A practical guide to wavelet analysis. Amer. Meteor. Soc., 79(1):61-78, 1998.

[11] A. Grinsted, J. C. Moore, and S. Jevrejeva. Application of the cross wavelet transform and wavelet coherence to geophysical time series. Nonlinear Proc. Geophys., 11:561-566, 2004.

[12] J. A. Schulte. Wavelet analysis for non-stationary, non-linear time series. Nonlinear Proc. Geophys., 23:257-267, 2016.

[13] D. E. Newland. An Introduction to Random Vibrations, Spectral \& Wavelet Analysis. Longman, Harlow, UK, 1993.

[14] M. J. de C. Henshaw. M219 cavity case: in, verification and validation data for computational unsteady aerodynamics. Tech. Rep. RTO-TR-26 AC/323(AVT)TP/19, Research and Technology Organization, Neuilly-Sur-Seine Cedex, France, October 2000.

[15] J. Basley, L. R. Pastur, F. Lusseyran, J. Soria, and N. Delprat. On the modulating effect of three-dimensional instabilities in open cavity flows. J. Fluid Mech., 759:546-578, 2014. 